\title{
A008
}

\section{Valuable Dusters - Not an Oxymoron}

\section{I.M. Longley* (GIS-pax)}

\section{SUMMARY}

There is no common standard for exploration well failure analysis within the broader upstream industry. Often dry wells are simply ignored; even when they are studied the analysis methodology that is performed varies by company and sometimes even within a company. Typically all these methods are documented as static records

in spreadsheets or reports which are not updated with new or changing information and data. 
There is no common standard for exploration well failure analysis within the broader upstream industry. Often dry wells are simply ignored; even when they are studied the analysis methodology that is performed varies by company and sometimes even within a company. Typically all these methods are documented as static records in spreadsheets or reports which are not updated with new or changing information and data.

A structured approach is described in this presentation where what is known (or not known) about the play elements (trap, reservoir, seal and charge) for different individual prospect targets within a well is captured systematically and these data are then integrated to classify the target result into one of the following hierarchical test types:

1. A discovery - where all the elements are present and effective.

2. A dry valid trap test - where all the elements except charge are present.

3. A failed trap test - where reservoir or containment elements (top, base or fault seal elements) have failed.

4. A low confidence trap test where there is some uncertainty as to whether a valid trap has been drilled or not.

5. An off structure test where no valid trap configuration is interpreted to have been present.

6. An unevaluated test where at least one of the play elements is unevaluated.

Results from this analysis (success rates, failure modes) can be used to calibrate and constrain play map evaluations and to aid and spatially constrain prospect chance evaluations. Analog populations are sampled spatially, stratigraphically and/or by geological trap type.

The methodology is simple and powerful and easy to keep current as new data becomes available. It also serves as an audit trail for technical evaluations and a knowledge capture mechanism for valuable information that is typically lost when technical people leave a company. 